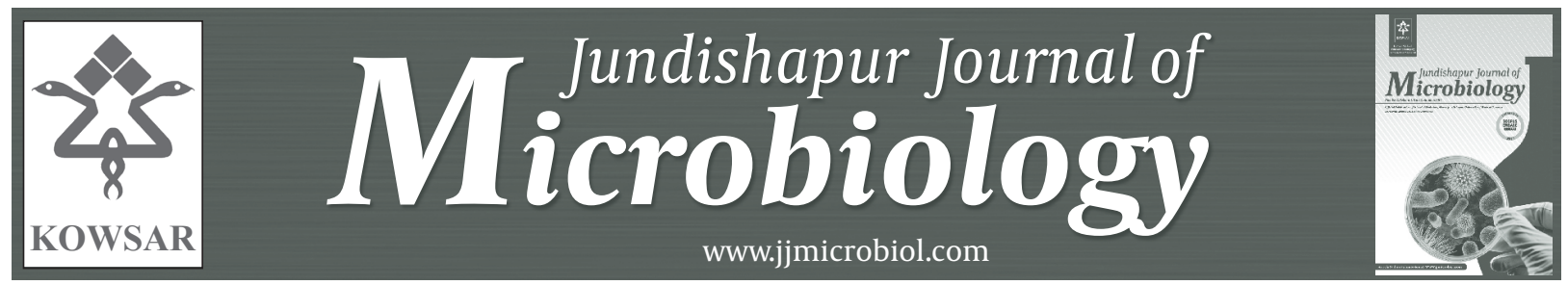

\title{
Study of the Assimilation Rate of Immunoenzymatic Tests and Traditional Serological Methods in the Diagnosis of Human Brucellosis
}

\author{
Homayoon Bashiri ${ }^{1}$, Babak Sayad ${ }^{1}$, Seyed Hamid Madani ${ }^{1, *}$ \\ ${ }^{1}$ Molecular Pathology Research Center, Imam Reza Hospital, Kermanshah University of Medical Sciences, Kermanshah, IR Iran \\ *Corresponding author: Seyed Hamid Madani, Molecular Pathology Research Center, Imam Reza Hospital Kermanshah University of Medical Sciences, \\ Kermanshah, IR Iran., E-mail: shmmadani@yahoo.com.
}

\begin{abstract}
A B S T R A C T
Background: Brucellosis is a disease carried by animals that can be transmitted to humans. The signs and symptoms of brucellosis are nonspecific, blood tests and blood/tissue cultures are necessary for making the diagnosis of brucellosis. Testing for antibodies against the bacteria and isolating the organism from blood cultures and biopsy of body tissue (from the bone marrow or the liver) are various methods of diagnosis of brucellosis. In the absence of bacteriologic confirmation, a presumptive diagnosis can be made on the basis of high or rising titers of specific antibodies.

Objectives: It is observed practically, that the sensitivity of serological tests is less than the amounts mentioned in the reference books. Elisa is a new method for diagnosis of the disease and in this study; application and its assimilation rate are compared with traditional serological tests.

Materials and Methods: In this study, patients were selected who had suspicious clinical symptoms of brucellosis. Serological and Elisa tests were performed simultaneously.

Results: In this setting, detection rate of the disease is $34.86 \%$, if the high normal titer for the Wrights test titer assumed $1 / 80$ and is $44.95 \%$ if the titer assumed 1/40, while Elisa test had detection rate of 58.72\% for those suspicious cases. Furthermore, the detection rate for diagnosis of active brucellosis with $2 \mathrm{ME}$ tests was $17.54 \%$ with high normal titer of $1 / 80$ and was $59.64 \%$ with a high normal titer of $1 / 40$.

Conclusions: The use of the Elisa tests is preferable for the diagnosis, and if the serological tests are used it is better that the high normal titer assumed to be $1 / 40$.
\end{abstract}

Keywords: Brucellosis; Elisa Test; Traditional Serological Test

Copyright @ 2013, Ahvaz Jundishapur University of Medical Sciences; Published by Kowsar Corp.

Article type: Research Article; Received: 29 Apr 2012; Revised: 18 Aug 2012; Accepted: 03 Sep 2012; Epub: 01 Jun 2013 ; Ppub: Jun 2013

Implication for health policy/practice/research/medical education:

According to this objectivity that Brucellosis is an endemic disease in some parts of IRAN, researches about more accurate laboratory tests are useful for better diagnosis of this disease.

-Please cite this paper as:

Bashiri H, Sayad B, Madani SH. Study of the Assimilation Rate of Immunoenzymatic Tests and Traditional Serological Methods in the Diagnosis of Human Brucellosis. Jundishapur J Microbiol. 2013; 6(4):e4828. DOI: 10.5812/jjm.4828

Copyright @ 2013, Ahvaz Jundishapur University of Medical Sciences; Published by Kowsar Corp.

This is an Open Access article distributed under the terms of the Creative Commons Attribution License (http://creativecommons.org/licenses/by/3.0), which permits unrestricted use, distribution, and reproduction in any medium, provided the original work is properly cited. 


\section{Background}

A. Brucellosis is a commonly transmitted disease between human and animals (1), which is usually transferred to man from infected animals. This disease is caused by one of the four subtypes of Brucella, Brucella melitensis (the most common), B. abortus, B. suis and $B$. canis. Its global prevalence is not exactly known due to lack of the uniform information systems and the lack of reports on the disease in many countries (2). Since the symptoms of the disease are nonspecific and isolation of the organism is very difficult, diagnosis is established using serological methods (3). These methods consist of various tests including : Wright test which assess IgM and IgG, Coombs Wright test which assess IgG, 2ME test which assess IgG,and immunoenzymatic test, consisting of Elisa test which can assess IgM or IgG, individually (4). Rose Bengal test is a screening test only, and requires confirmation with another test in positive cases (5).

Wright test is the most commonly used test, and it claims to be able to diagnose as many as $>97 \%$ of cases of brucellosis. Reference titers of $1 / 640$ or more, and or an increase of four times in the titer of antibodies are considered positive for this test in textbooks. However, for some reason in endemic countries like Iran, a titer of $1 / 160$ or more has been proposed for diagnosis of the patients. Of course, there are false positive and false negative results.

$2 \mathrm{ME}$ is a diagnostic test to identify cases requiring treatment, ie, active brucellosis. Generally, titer of 1/160 or more are considered positive for this test. Titer lower than $1 / 80$ and $1 / 40$ are rare and under special circumstances are indicative of recent significant infections (6, 7). For these purpose traditional serological tests such as Wright, 2ME and Coombs Wright tests are used in Iran. Although they are claimed to posses high sensitivity and specificity, they have some limitations, as well (8).

It has been detected by experience and reported in some studies that their sensitivity and specificity are not as high as claimed in their references $(4,9)$. For this reason, we decided to compare the diagnostic value of these traditional serological tests with more modern ones like Elisa. Its' sensitivity for Brucella has been reported as 96 to98.2\% in different studies, and its specificity as 98.8 to $100 \%(8,10)$. Meanwhile several studies recommend simultaneous use of these traditional and modern serological tests (11).

\section{Objectives}

The main objective of this study is to compare two methods of laboratory diagnosis of brucellosis, ie, traditional serological tests and Elisa test.

\section{Materials and Methods}

In this study, upon approval of a specialist for infectious diseases, patients were selected with clinical manifestations suggesting brucellosis. The patients were having the symptoms for up to two weeks. Traditional serological tests (Wright and 2ME) and immunoenzymatic tests (Elisa including IgG and IgM) were performed for all the patients at the same time. All the tests were carried out in a licensed medical laboratory by licensed technicians. Considering sensitivity of $98 \%$, accuracy of $93 \%$ and degree of certainty of $95 \%$, the minimum number of cases was decided to be 84 .

Regarding the length of the study (2.5 years), 109 patients were chosen, and the statistical data of all the patients were used to enhance the levels of certainty and accuracy. To perform the tests, 2-4 $\mathrm{ml}$ of blood was drawn from each patient in EDTA anticoagulant, and the Wright test was performed using tubal standard method with 10 test tubes. To perform the immunonzymic test of Elisa, the Trinity kit of Biotech Company were used. Each of the anti Brucella antibodies of IgG and IgM were measured separately.

After performing and recording all the tests results, at first the Wright test was compared to the Elisa test (IgM or IgG). Since the $2 \mathrm{ME}$ test only assesses IgG, the second comparison was made between $2 \mathrm{ME}$ and Elisa IgG. For the Wright test, titers of $1 / 80$ or higher were considered as positive (5). For 2ME test, titers of 1/80 and higher (2) were considered positive (6). For Elisa test, a titer of more than 1.1 was considered positive and a titer of less than 0.9 as negative. Any values between these two were considered as doubtful. In our study, 3 cases were identified as doubtful, and they were detected as negative with regards to their serological testes. All three were discarded from the study ( Tables 1,2 ).

Table 1. The Relationship between Positive Blood Cultures and Titer of Wright Test in Three Studies in Iran

\begin{tabular}{llll|}
\hline Imam Hospital & Razi Instate & $\begin{array}{l}\text { Loghman } \\
\text { Hospital }\end{array}$ & Wright Titer \\
\hline $\mathbf{7} \%$ & $1 \%$ & 0 & 0 \\
\hline $\mathbf{0}$ & 0 & 0 & $1 / 10$ \\
\hline $\mathbf{0}$ & $7 \%$ & 0 & $1 / 20$ \\
\hline $\mathbf{0}$ & $9 \%$ & $17 \%$ & $1 / 40$ \\
$\mathbf{6} \%$ & $7 \%$ & $8 \%$ & $1 / 80$ \\
$\mathbf{1 2} \%$ & $22 \%$ & $17 \%$ & $1 / 160$ \\
\hline $\mathbf{3 0} \%$ & $25 \%$ & $24 \%$ & $1 / 320$ \\
\hline
\end{tabular}


Table 2. The Relationship Between Positive Blood Culture and Titer of 2ME Test in Two Studies in Iran.

\begin{tabular}{lll}
\hline \multicolumn{3}{c}{ Positive Blood Culture } \\
\hline Loghman Hospital & Razi Institute & 2ME Test Titer \\
\hline $\mathbf{0}$ & $19 \%$ & 0 \\
$\mathbf{8 . 2} \%$ & $5 \%$ & $1 / 10$ \\
$\mathbf{1 6 . 6} \%$ & $12 \%$ & $1 / 20$ \\
$\mathbf{1 6 . 7} \%$ & $17 \%$ & $1 / 40$ \\
$\mathbf{1 6 . 7} \%$ & $23 \%$ & $1 / 80$ \\
$\mathbf{2 5} \%$ & $14 \%$ & $1 / 160$ \\
$\mathbf{1 6 . 7} \%$ & $9 \%$ & $1 / 320$ \\
$\mathbf{0}$ & 0 & $1 / 640$ \\
$\mathbf{0}$ & $1 \%$ & $1 / 1280$ \\
$\mathbf{0}$ & $1 \%$ & $1 / 2560$ \\
$\mathbf{1 0 0} \%$ & $100 \%$ & Total \\
\hline
\end{tabular}

\section{Results}

Out of the 109 patients, 29 (26.6\%) were male with the average age of 36.6 , and 80 (73.4\%) were female with the average age of 42.7. The youngest was a 7 year old girl and the oldest a 77 year old man.

The comparison of the two methods was performed for patients with a titer of 1/80 or higher of traditional tests and Elisa (IgM or IgG). Out of the 109 patients suspected for brucellosis based on their clinical manifestations, 64 patients (58.7\%) had a positive Elisa test, while the Wright test are positive only in 38 cases as positives (34.9\%). Evaluating these findings using McNamara test revealed no correlation between the two methods ( Table 3 ).

Table 3. Evaluating Wright Test and Elisa-IgM Test Using McNamara Test (Wright Titer of $1 / 80$ or Higher as Positive)

\begin{tabular}{lllll}
\hline & & \multicolumn{3}{c}{ Wright Test } \\
\cline { 3 - 5 } & & + & - & Total \\
\hline Elisa- IgM Test & + & 37 & 27 & 64 \\
& - & & 24 & 45 \\
& Total & 38 & 71 & 109 \\
\cline { 2 - 5 }
\end{tabular}

Table 4. Evaluating Elisa- IgG and 2ME Based on McNamara Test (2ME $>1 / 80$ as Positive)

\begin{tabular}{|lllll}
\hline & & \multicolumn{3}{l}{ Wright Test } \\
\cline { 3 - 5 } & & + & - & Total \\
\hline Elisa- IgG Test & + & 19 & 54 & 73 \\
& - & 0 & 36 & 36 \\
& Total & 19 & 90 & 109 \\
\cline { 2 - 5 }
\end{tabular}

A comparison of $2 \mathrm{ME}$ (in cases with titer of $1 / 80$ and higher) and IgG-Elisa was performed, as well. Out of 109 patients suspected for brucellosis based on their clini- cal manifestation, 73 patients $(66.9 \%)$ had positive IgG Elisa test results, while $2 \mathrm{ME}$ test detected only 19 patients (17.4\%) as positive. Evaluating these results using McNammara test showed that no correlation actually existed between the two (Table 4).

In the next step, a comparison between Wright test (in cases with titer of 1/80 and higher) and Elisa (IgM and IgG) was performed. Out of the 109 patients, 64 (58.7\%) had positive Elisa test results, and only $45(41.3 \%)$ had positive Wright test results. Evaluating these results using McNamara test revealed no significant correlation between the two methods (Table 5).

A comparison between $2 \mathrm{ME}$ test (when titer of higher than 1/40 was considered positive) and Elisa-IgG was performed, as well. Out of the 109 patients, 73 (67\%) were detected as positive by Elisa-IgG test, while only 47 patients (43.9\%) were $2 \mathrm{ME}$ positive. Evaluating these results by McNamara test showed no correlation between the two methods (Table 6). According to the above- mentioned results, the importance of early diagnosis to prevent future complications, along with the prolonged culture period (at least 4 weeks) or limited occurrence of bacteremia (15$70 \%$ ) is of paramount importance. The importance of culture for rapid diagnosis has received little attention for clinical purposes in many cases ( 2 ). As a result, the routine diagnostic procedure consists of the appearances of serum antibodies.

Table 5. Evaluating Wright and Elisa-IgM Test Based on McNamara Test (Wright $>1 / 80$ as Positive)

\begin{tabular}{|lllll|}
\hline & & \multicolumn{3}{c|}{ Wright Test } \\
\cline { 2 - 5 } & & + & - & Total \\
\hline Elisa- IgM Test & + & 48 & 16 & 64 \\
& - & 1 & 44 & 45 \\
& Total & 49 & 60 & 109 \\
\cline { 2 - 5 } &
\end{tabular}

Table 6. Evaluating 2ME Test and Elisa- IgG Based on McNamara Test (2ME $>1 / 40$ as Positive)

\begin{tabular}{lllll}
\hline & & \multicolumn{3}{c}{ 2ME Test } \\
\cline { 3 - 5 } & & + & - & Total \\
\hline Elisa- IgM Test & + & 47 & 26 & 73 \\
& - & 0 & 36 & 36 \\
& Total & 47 & 72 & 109 \\
\cline { 2 - 4 }
\end{tabular}

\section{Discussion}

In order to find a simple and accurate method, many studies have been performed on comparing diagnostic methods, for diagnosis of brucellosis. Elisa test is a relatively new one diagnostic test has been studied in several studies. Its specificity and sensitivity has been reported as high as $100 \%(6-8,10)$.

For serologic tests, sensitivity of $97 \%$ has been reported 
(4). However, as it has been observed and even reported in some studies, that many patients present with suspected clinical manifestations of brucellosis and have responded positively to treatment however, their serologic tested negative. In Loghman Hospital in Tehran, $40 \%$ of patients with positive cultures have $2 \mathrm{ME}$ titer of 1/40 or lower. Similarly, Razi Institute reported that $17 \%$ of patients having positive culture revealing Wright test titer of lower than $1 / 80$. Some studies have also shown the priority of Elisa tests to serological ones (10-12), and some others have limited the use of Elisa to certain subtypes of brucellosis (13).

With regards to the positive titer of Wright and $2 \mathrm{ME}$ tests, which was reported as 1/160 in reference books and as 1/80 in local references of developing countries like Iran $(4,14)$, a significant difference between positive cases of Elisa tests and traditional serological ones are observed. Even if a titer of lower than $1 / 40$ is chosen, the difference is still significant. If the positive titers were considered as $1 / 80$, Elisa test would report $58.7 \%$ of suspected cases as positive while Wright test would report only $34.9 \%$ as positive. A positive titer of $1 / 40$ would yield $58.7 \%$ of suspected cases as positive with Elisa test and only 41.3\% with Wright test. This shows that using Elisa test has decreased false negative results of diagnostic tests. This has also been found to be statistically significant. A comparison of 2ME and Elisa-IgG has revealed similar results. Interestingly, no patient with $2 \mathrm{ME}$ titer of $1 / 40$ was found to be Elisa-IgG negative.

Considering the above-mentioned data and the results of the present study, it is suggested that in suspicious or negative serological tests, with strong clinical suspicion for brucellosis, Elisa tests can be recommended as the next step (15). In addition, when using serological tests, we should be more cautions with our interpretation and not simply discard patients with a serological titer of $1 / 40$. If we strongly suspect the disease clinically, we had best to take steps to diagnosis the disease, as well as commencing treatment of the disease.

\section{Acknowledgements}

We thank Kermanshah University of Medical Sciences for funding of all expenses of this research.

\section{Authors' Contribution}

None declared.

\section{Financial Disclosure}

None declared.

\section{Funding/Support}

None declared.

\section{References}

1. Spink WW. Nature of brucellosis. 1956.

2. Corbel MJ. Brucellosis: epidemiology and prevalence worldwide. 1989.

3. Young EJ. Serologic diagnosis of human brucellosis: analysis of 214 cases by agglutination tests and review of the literature. Rev Infect Dis. 1991;13(3):359-372.

4. Young EJ. Immunology of brucellosis. Madkour's Brucellosis. 2nd ed. Berlin, Germany: Springer-Verlag. 2001:39-50.

5. Hatami H. [Clinical epidemiology and bioterrorism related diseases control, Center for disease control of Iran]. 2002.

6. Araj GF. Update on laboratory diagnosis of human brucellosis Int J Antimicrob Agents. 2010;36 Suppl 1:S12-7.

7. Mantecon Mde L, Gutierrez MP, Zarzosa Mdel P, Fernandez-Lago $\mathrm{L}$, Colmenero Jde D, Vizcaino N, et al. Influence of brucellosis history on serological diagnosis and evolution of patients with acute brucellosis. J Infect. 2008;57(5):397-403.

8. Osoba AO, Balkhy H, Memish Z, Khan MY, Al-Thagafi A, Al Shareef $\mathrm{B}$, et al. Diagnostic value of Brucella ELISA IgG and IgM in bacteremic and non-bacteremic patients with brucellosis.J Chemother. 2001;13 Suppl 1:54-9.

9. Pakzad P. [Principles of clinical serological tests].1996.

10. Renukaradhya GJ, Isloor S, Crowther JR, Robinson M, Rajasekhar M. Development and field validation of an avidin-biotin enzymelinked immunosorbent assay kit for bovine brucellosis. Rev Sci Tech. 2001;20(3):749-56.

11. Amirzargar A. comparison of diagnostic and bacteriologic tests of brucellosis in Ahwaz province of Iran. J Yasoj Medical Faculty. 2001;21-22:60-63.

12. Karbasian A. [Application of immunoenzymatic reactions in the diagnosis of human brucellosis]. J Isfahan Medical Faculty:;1:19-24

13. Lisik D, Sobieszczanska B. [Immunoenzymatic test ELISA in serodiagnosis of chronic brucellosis]. Przegl Epidemiol 2001;55(3):299-303.

14. Mandell GL, Bennett JE, Dolin R. Principles and practice of Infectious diseases. 2005.

15. Grushina T, Atshabar B, Syzdykov M, Daulbaeva S, Tserelson L, Kuznetsov A, et al. Universal indirect enzyme-linked immunosorbent assay for monitoring of human and animal brucellosis in Kazakhstan. Vaccine. 2010;28 Suppl 5:F46-8. 